\title{
Introducing novel light management to design a hybrid high concentration photovoltaic/water splitting system
}

\author{
José JH Videira ${ }^{1}$, Keith WJ Barnham ${ }^{1}$, Anna Hankin ${ }^{2}$, James P Connolly ${ }^{3}$, Matthew Leak ${ }^{1}$, Jonathan Johnson ${ }^{1}$, \\ Geoff H Kelsall ${ }^{2}$, Ken Kennedy ${ }^{4}$, John S. Roberts ${ }^{4}$ and Amanda J Chatten ${ }^{1}$ \\ ${ }^{1}$ Department of Physics, Imperial College London, London, UK \\ ${ }^{2}$ Department of Chemical Engineering, Imperial College London, London, UK \\ ${ }^{3}$ Nanophotonics Technology Centre, Universidad Politecnica de Valencia, Valencia, Spain \\ ${ }^{4}$ EPSERC National Centre for III-V Technologies, University of Sheffield, UK
}

\begin{abstract}
We present a novel way to utilize high-concentration photovoltaic (HCPV) radiative losses and diffuse light, otherwise unused in conventional HCPV systems, to power an Imperial College designed photoelectrochemical reactor (PECR) producing $\mathrm{H}_{2}$ fuel through water splitting. A high efficiency photovoltaic (HEPV) is embedded inside a Luminescent Solar Concentrator (LSC). Edge emission from the radiative recombination loss mechanism in the HEPV is guided within the LSC to the PECR photocathode, whilst the LSC emitted light is guided to the photoanode. The photon streams can be independently optimised in intensity and wavelength. We demonstrate how photon streams with balanced intensity can be achieved.
\end{abstract}

Index Terms-geometrical optics, luminescent devices, photoluminescence, quantum wells, ray tracing, solar cells.

\section{INTRODUCTION}

The extraction of hydrogen from water using sunlight was first proposed 30 years ago [1]. Recently interest in the subject is fueled by the need to find a renewable, sustainable and environmentally safe alternative form of energy storage. The main challenge in this field of research is finding a material that can match the energetic and stability requirements of spontaneous photo-water splitting [2], and a breakthrough in this would be the surest way to reach efficiencies high enough to seriously consider commercialisation [3].

The ultimate aim of this project, in conjunction with the Chemical Engineering department at Imperial College London, is to demonstrate photoelectrochemical (PEC) water splitting using photoanodes and photocathodes (Figure 1) that are independently illuminated by two photon streams that are conventionally regarded as waste from HCPV operation. A further advantage of the scheme is that the intensity and wavelength of each photon stream can be independently optimised. The system would produce zero-carbon hydrogen for energy storage and transport as a by-product of HCPV electrical power generation. These two waste photon streams are 1) light that is emitted due to dominant radiative recombination mechanisms at high cell operating bias and 2) entirely unused diffuse light.

We present a concept that merges conventional CPV and LSC technology into a hybrid system that can both generate electricity and generate hydrogen through PEC water splitting.

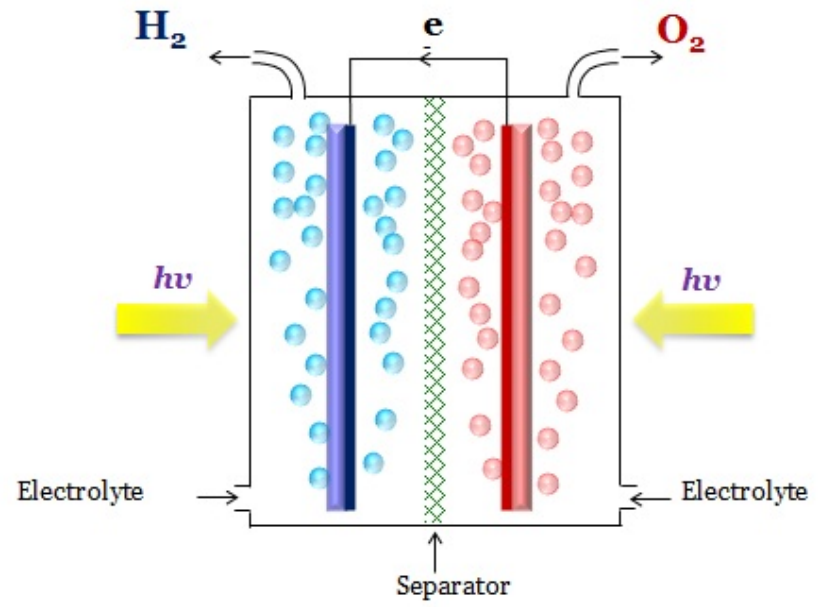

Fig. 1. Schematic of a doubly illuminated photoelectrochemical reactor (PECR). The photoanode (red) produces oxygen molecules through oxidation of water whilst the photocathods (blue) produces hydrogen molecules through the reduction of hydrogen ions.

\section{A System OvervieW}

A diagram of the system is shown in Figure 2. The challenge of directing two separate photon streams to individual photoelectrodes can be solved with a modified luminescent solar concentrator (LSC). The LSC, first introduced in the 1970s, in its basic design is a transparent waveguide structure, either homogeneously doped with, or coated with, luminescent material (luminophores). The luminophores absorb incident light at or above their absorption threshold, and re-emit it with a certain redshift with ideally near unity luminescent quantum yield (LQY). A large fraction of this emitted light is waveguided by total internal reflection (TIR) to the structure's edges, where it is collected by optically coupled optoelectronic devices. The main benefit of using a LSC is the ability to tune its absorption/emission spectra according to different needs, by using different luminophore species. The LSC will provide one of the photon streams by absorbing diffuse light, re-emitting it and waveguiding it into the photoanode of the PECR.

For the other photon stream, we will use high efficiency photovoltaics (HEPV). HCPV modules utilise low-cost optical elements to reduce the amount of semiconductor needed by 
concentrating direct sunlight onto these very small but highefficiency cells. This replacement of expensive semiconductor with cheaper optics provides a route towards reducing the costs of solar electricity generation. A conventional HCPV module consists of three elements: The concentrating optics, the solar cells and the housing. The aim is to concentrate light as much as possible, onto a HEPV, and to dissipate heat as much as possible.

Any high quality HEPV at high cell operating bias will experience radiatively dominated recombination losses. Therefore at its maximum power point, when forward biased by the absorption of concentrated sunlight, a HEPV can act like an edge emitting LED. However, quantum well solar cells (QWSCs) are particularly suitable for this application for three reasons: 1) As will be discussed, their radiative efficiency can be simply established from measurements of quantum efficiency and dark current. 2) The radiative recombination takes place at the bottom of the quantum well. Quantum wells are a common feature of LEDs because of their high radiative efficiency and because the barrier and substrate materialsl is transparent to the radiation. 3) The wavelength and intensity of the emission can be optimised for a given catalyst or photoelectrode simply bythe choice of well depth.

The HEPV, which will have antireflection coating on its edges, is embedded in and optically coupled to, an undoped waveguide which forms the transparent substrate of a square LSC with thin-film luminescent coating on the top surface, as in Figure 2.

A Fresnel lens concentrates direct light onto a quantum well solar cell (QWSC) to generate a photocurrent, as per conventional HCPV functioning. Depending on the QWSC material structure and operating conditions, a certain fraction of the light from radiative recombination will be waveguided to the edges and be coupled into the LSC, where it gets trapped due to total internal reflection, and waveguided to the photocathode. The majority of the edge emission from the QWSC will be at an angle greater than the escape cone from the top and bottom surfaces of the waveguide [4]. The emission will also be longer wavelength that the luminescence from the LSC coating. Hence the thin-film coating of the LSC will not absorb the radiation from the QWSC.

In addition to the essentially isotropic diffuse contribution of light to the LSC, there is also a contribution from the circumsolar sunlight due to the scattering of sunlight by atmospheric aerosols. The intensity of the short-wavelength photon stream can therefore be optimised for given spectral conditions by adjusting the acceptance angle of the CPV system.

Wavelength selective mirrors, which are coupled in between the LSC and the photoelectrodes, provide the means to direct the two photon streams to their desired locations. The photoanode, which in our scheme is illuminated by short wavelength light is coupled to the waveguide through a filter that transmits light in the visible range, and reflects it in the infrared range. The photocathode which on our scheme absorbs infrared light is coupled to the waveguide by a filter that transmits light in

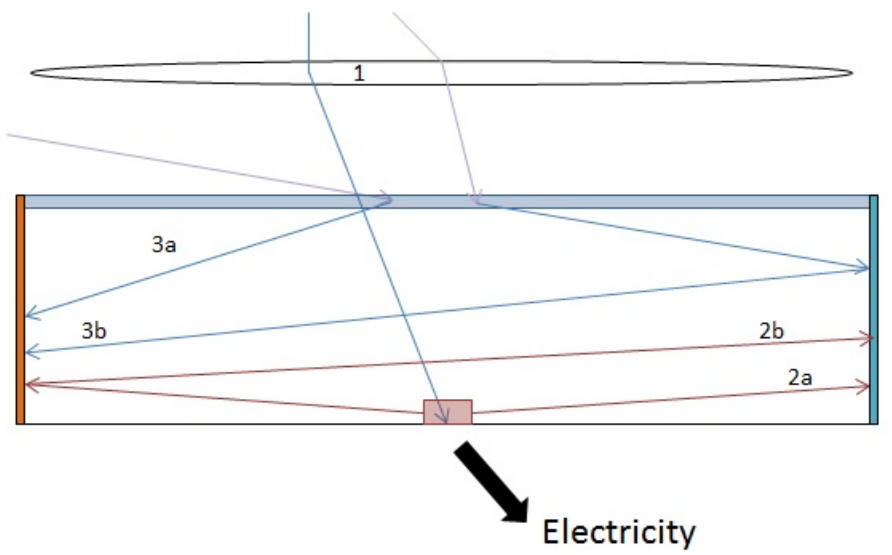

Fig. 2. Summary of the hybrid system optics. 1) Direct light is concentrated onto a high efficiency PV cell by Fresnel lens. 2) Emission from radiative recombination escapes from the PV and is waveguided into the reactor photocathode by (a) direct from PV edge, or (b) via hot mirror reflection. 3) Diffuse light is absorbed, re-emitted and waveguided into the reactor photoanode a) directly from luminescent re-emission, or b) via cold mirror reflection.

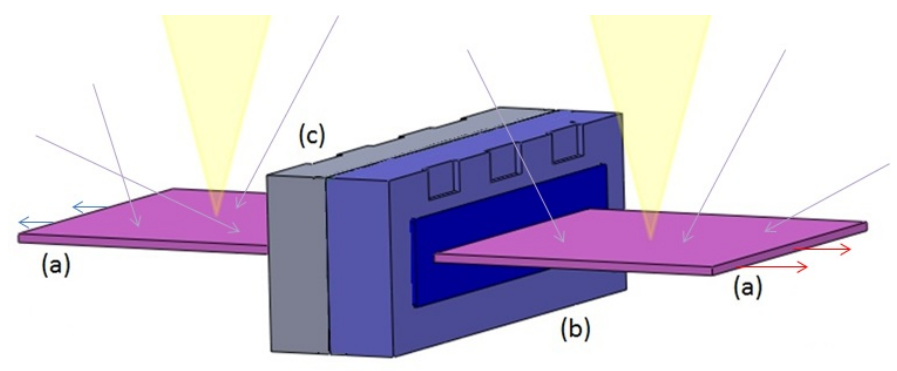

Fig. 3. Overview of the hybrid system, with a high efficiency photovoltaic (HEPV) embedded within a luminescent solar concentrator (LSC) (a). Concentrated direct light enters the solar cell, and UV/blue diffuse light gets absorbed by the LSC. The resultant emitted light from both the LSC and the QWSC gets waveguided into both the photoanode (b) and photocathode (c) sections of the PECR respectively. Note the modularity of the system, where an array of LSCs/PECRs is possible.

the infrared and reflects it in the visible range. The two other edges as well as the underside of the LSC have Lambertian high reflectivity air-gap reflectors to minimise escape losses.

The wavelengths of the photon streams are engineered to be appropriate for the electrode materials thus allowing the water splitting half reactions that produce $\mathrm{H}_{2}$ to occur within the PECRs. As shown in Figure 3, the system is designed to be modular, with a view of creating a series of arrays of LSCs and PECRs. Figure 4 shows how the reflectivity spectra for the mirrors correspond well with the spectra for both photon streams.

\section{Testing The PRINCIPLE OF THE QuANTUM Well SOLAR CELl aCTING as LUMINESCENT SOLAR CONCENTRATOR}

\section{A. Premise}

The starting point of system optimisation is the initial calculation of the power density that can be achieved by the LSC and the HEPV. In this section we present the calculations 


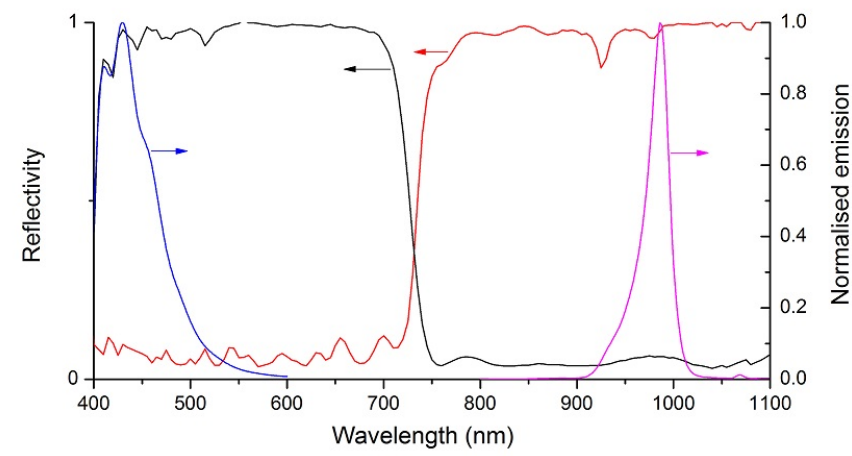

Fig. 4. Reflectivity of the cold (black) and hot (red) mirror as a function of wavelength at $0^{\circ}$ angle of incidence. The LSC emitted light (blue) gets transmitted by the hot mirror and reflected by the cold mirror, whereas the opposite happens for the HEPV edge emission (magenta).

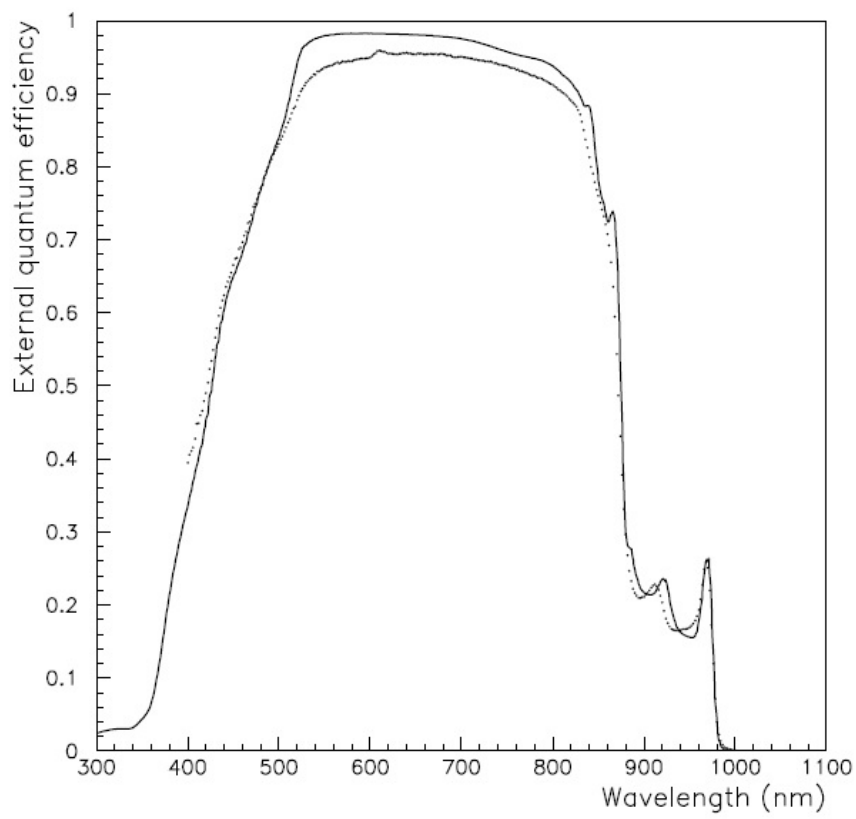

Fig. 5. Measured and fitted external quantum efficiency (EQE) of a p-i-n strain-balanced quantum well solar cell, with its dark current in Figure 6

for both power densities when the system is illuminated under ASTM G-173 conditions [5] and the HEPV is being operated at $500 \mathrm{x}$ concentration.

\section{B. Luminescent Solar Concentrator}

For our calculations we use a thin-film luminescent solar concentrator (LSC), with a luminophore/poly(methyl methacrylate) (PMMA) film blade coated onto a UVtransparent glass substrate. The photoanode absorbs light at wavelengths shorter than $500 \mathrm{~nm}$. Therefore we utilise Lumogen F Violet 570 (LV570), a naphthalimide-based luminophore. It absorbs light in the $300 \mathrm{~nm}-410 \mathrm{~nm}$ region, and emits in the $390 \mathrm{~nm}-550 \mathrm{~nm}$ region with a luminescent quantum yield of around $85 \%$. The optical concentration $\mathrm{C}_{\mathrm{opt}}$, defined as the ratio of photon flux coupled from the edges to the

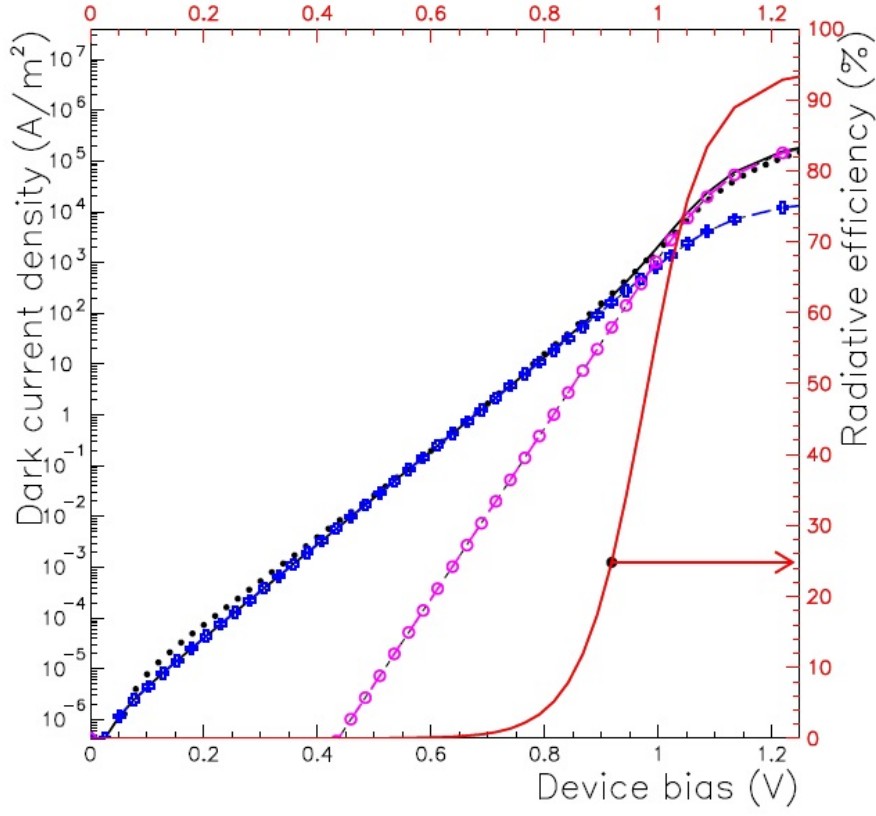

Fig. 6. Measured (black dots) and fitted (black line) dark-IV curve of a p-i-n strain balanced quantum well solar cell. The radiative contribution (magenta) is a prediction based on the quantum well absorption aobratined from the quantum efficiency fit in Figure 5 The SRH contribution (blue) is a one parameter fit

incident photon flux, can be manipulated by changing the geometry of the LSC. We use a combination of our in-house raytracer PVTrace [6] and the SMARTS sky spectral irradiance calculator [7] to give performance characteristics of the LSC in ASTM G-173 conditions.

The LV570 luminophore is mixed with PMMA at a dye load concentration of $1.5 \%$ and given dimensions of $200 \mathrm{x}$ $200 \times 5 \mathrm{~mm}$. With PVTrace we simulated the performance of this LSC setup under incident light conditions of an isotropic hemispherical light source with the spectrum of the diffuse component of ASTM G-173. The calculated optical efficiency of this setup was $2 \%$, giving a photon output of $1.9 \times 10^{17} \mathrm{~s}^{-1}$.

\section{Quantum Well Solar Cell}

QWSCs, first invented by Barnham and Duggan [8], offer an way to improve multi-junction solar cells by the ability to tune their bandgaps, as well as enhancing photon coupling due to recombination being dominated by radiative processes at high concentration (in excess of 200suns) [9] - [11].

We demonstrate the principle of a QWSC acting as an LED with a number of square photoluminescence samples of different sizes from a wafer QT1604 grown by MOVPE at the EPSRC National Centre for III-V Technologies at Sheffield. It consists of 22 strain-balanced GaAsP/InGaAs quantum wells in a GaAs p-i-n structure. There was an AlGaAs window below the p-doped GaAs emitter layer that can produce some limited transverse waveguiding due to its comparatively low refractive index. The edges of the photoluminescence sample were anti-reflection coated. 
In order to determine the power density coming out of the edges of the device when illuminated with 500x concentration, we must determine a) its radiative efficiency for a given incident power density and b) its waveguiding capabilities. This is done by a combination of modelling and edge photoluminescence measurements.

The radiative efficiency of a given QWSC can be determined by fits to the quantum efficiency and dark current as demonstrated in Figure 5 and Figure 6, with a fitting model, SOL [12]. The radiative efficiency is expressed as:

$$
\eta_{\text {rad }}=\frac{J_{\text {rad }}}{J_{\text {rad }}+J_{S R H}}
$$

Where $\mathrm{J}_{\text {rad }}$ is the radiative recombination current contribution and $\mathrm{J}_{\mathrm{SRH}}$ is the Shockley-Read-Hall non-radiative recombination current contribution. This efficiency is demonstrated in Figure 6 as the red line. The low bias dark current is fitted by Shockley-Read-Hall recombination with one parameter: the effective lifetime for carrier trapping. The radiative contribution to the dark current (magenta circles in Figure 6), which results in photon emission from around the first exciton in Figure 5. is an absolute prediction based on detailed balance assuming photon absorption is given by the fit to the QE around the first exciton feature. This procedure is similar to the determination of the secondary emission from a luminescent species in a LSC.

The merit of using a non-metalised photoluminescence sample is that it functions under open-circuit voltage $\mathrm{V}_{\mathrm{OC}}$, and without the complication of series resistance that causes the dark-current to curve at high bias in Figure 6 . Under $\mathrm{V}_{\mathrm{OC}}$ conditions, the current $\mathrm{J}_{\mathrm{V}}$ in the cell is zero, therefore the short circuit current $\mathrm{J}_{\mathrm{SC}}$ equals the dark current $\mathrm{J}_{\mathrm{D}}$ due to the expression:

$$
J_{\mathrm{V}}=J_{S C}-J_{D}
$$

The short circuit current $\mathrm{J}_{\mathrm{SC}}$ is expressed as [4] :

$$
J_{\mathrm{SC}}=q\left(\eta \frac{P_{o p t}}{h \nu}\right)
$$

where $\mathrm{P}_{\mathrm{opt}}$ is the incident optical power density and $\eta$ is the quantum efficiency

At open circuit $\mathrm{J}_{\mathrm{SC}}=\mathrm{J}_{\mathrm{D}}$ and from Figure 6 the radiative efficiency and hence the radiative contribution to the dark current density can be determined. The radiative current through the cell is the product of this radiative current density and the sample area. The current is converted into a photon flux by dividing by the electronic charge. The photon flux at the cell edge depends on the cell waveguide efficiency $\eta_{\mathrm{C}}$. The photon flux at the PEC depends on the LSC waveguide efficiency at the wavelength of the emission.

QT1604 was uniformly illuminated with a $532 \mathrm{~nm}$ beam from a Millennia $\mathrm{V}$ laser with two different power settings. A shield blocked all incident and top surface PL light from entering the detector. A calibrated fibre spectrometer was coupled onto an edge of QT1604 to give an absolute spectrum in units of $\mu \mathrm{Wcm}^{-2} \mathrm{~nm}^{-1}$, shown in Figure 7 The curves were

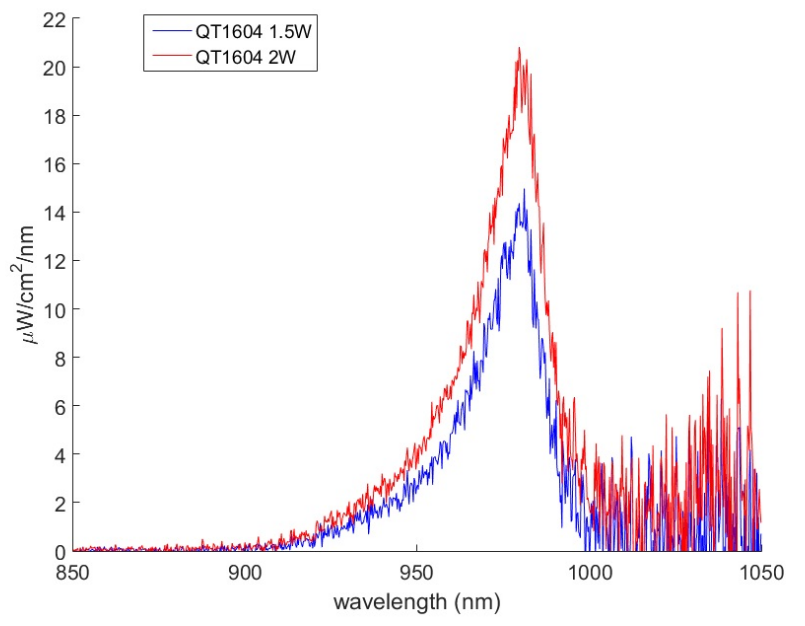

Fig. 7. Spectral irradiance of the edge emission from QT1604, a quantum well solar cell PL sample

fitted to Gaussian functions and integrated to give a value of power density leaving one of the edges. Multiplying up by the area of the edge of the sample we obtain the power out of the edge. To calculate the maximum photon flux coming out of the edges of the sample, we will use Equation 3 with the method mentioned previously.

Using the incident photon flux and the EQE of the sample, we calculate the dark current $\mathrm{J}_{\mathrm{D}}$. Using the radiative efficiency $\eta_{\mathrm{C}}$ we obtain a radiative current $\mathrm{J}_{\mathrm{rad}}$. By dividing by charge $\mathrm{q}$ and the area of the edge of the sample we obtain a maximum edge photon flux out of the edge. Multiplying this value by four gives total maximum photon flux out of all edges of the sample.

Having the photon flux from the edge, and all the other parameters for Equation 1 and Equation 2 we can establish the waveguide efficiency of the sample:

TABLE I

Measuring the WAVEguide EFFICIEnCy of Photoluminesence SAMPLE QT1604

\begin{tabular}{lll} 
Photon flux incident on top surface $\left(\mathrm{m}^{-2} \mathrm{~s}^{-1}\right)$ & $2.04 \times 10^{23}$ & $2.74 \times 10^{23}$ \\
\hline $\mathrm{J}_{\mathrm{D}}\left(\mathrm{A} / \mathrm{m}^{2}\right)$ & $3.0 \times 10^{4}$ & $4.02 \times 10^{4}$ \\
Radiative efficiency & 0.85 & 0.86 \\
$\mathrm{~J}_{\text {rad }}\left(\mathrm{A} / \mathrm{m}^{2}\right)$ & $2.55 \times 10^{4}$ & $3.46 \times 10^{4}$ \\
$\mathrm{I}_{\mathrm{rad}}(\mathrm{A})$ & $6.37 \times 10^{-1}$ & $8.66 \times 10^{-1}$ \\
Maximum photon count $\left(\mathrm{s}^{-1}\right)$ & $3.98 \times 10^{18}$ & $5.35 \times 10^{18}$ \\
\hline Measured edge photon flux $\left(\mathrm{m}^{-2} \mathrm{~s}^{-1}\right)$ & $7.77 \times 10^{19}$ & $1.11 \times 10^{20}$ \\
Measured edge photon count $\left(\mathrm{s}^{-1}\right)$ & $7.75 \times 10^{14}$ & $1.10 \times 10^{15}$ \\
\hline Waveguide efficiency $\eta_{\mathrm{c}}(\%)$ & 0.02 & 0.02
\end{tabular}

Measuring QT1604 with dimensions $5 \mathrm{~mm} \times 5 \mathrm{~mm} \times 0.5 \mathrm{~mm}$ with two different laser power settings. The maximum photon flux is calculated using Equation 3 The radiative efficiency is 0.85 and the EQE is 0.92 at incident wavelength of $532 \mathrm{~nm}$.

Preliminary studies with a Monte Carlo model suggest this very small cell waveguiding efficiency is due to the free carrier absorption in the highly doped substrate. Though small, $\eta_{\mathrm{c}}$ can be used to calibrate the Monte Carlo model using the edge 


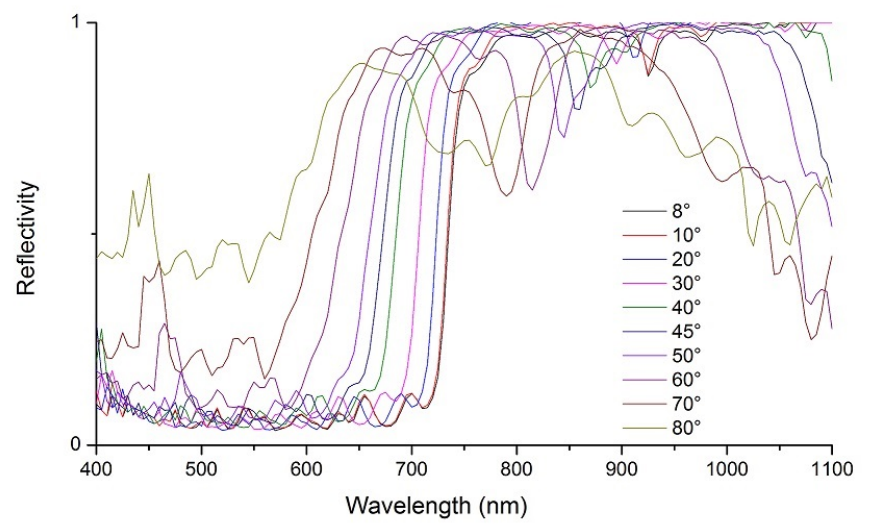

Fig. 8. Angular and wavelength dependence of the reflectivity of a hot mirror. Note the degradation of performance after the angle of incidence exceeds $60^{\circ}$.

measurements.

Using SOL, we can calculate the necessary QWSC waveguiding efficiency needed for ASTM G-173, 500x concentration light conditions when the QWSC is at maximum power point. An LSC with the parameters mentioned above under diffuse illumination will produce on the edges a photon count of $1.9 \times 10^{17} \mathrm{~s}^{-1}$.

Table II shows that this photon flux from the diffuse radiation absorbed by the LSC can be matched with the waste radiative emission from the QWSC even with the high resistance cell in Figure 6 as long as the cell waveguide efficiency is around $62 \%$. The second column is more typical of an optimised concentrator cell. Methods of reaching these cell waveguide efficiencies is discussed in section IV.

TABLE II

CALCULATING THE NECESSARY WAVEGUIDING EFFICIENCY OF QT1604

\begin{tabular}{lll} 
& High resistance & Low resistance \\
$\mathrm{J}_{\mathrm{SC}}$ at $500 \times$ concentration $\left(\mathrm{Am}^{-2}\right)$ & $1.28 \times 10^{5}$ & $1.28 \times 10^{5}$ \\
\hline Series resistance $(\Omega)$ & 0.22 & 0.03 \\
Voltage at MPP $(\mathrm{V})$ & 0.998 & 1.016 \\
$\mathrm{~J}_{\mathrm{rad}}$ at Vmpp $\left(\mathrm{Am}^{-2}\right)$ & 1103.25 & 2119 \\
$\mathrm{I}_{\mathrm{rad}}(\mathrm{A})$ & $5.41 \times 10^{-2}$ & $1.04 \times 10^{-2}$ \\
Max. Photon count $\left(\mathrm{s}^{-1}\right)$ & $3.38 \times 10^{17}$ & $6.49 \times 10^{17}$ \\
\hline LSC waveguide efficiency $(\%)$ & 90 & 90 \\
QWSC waveguide efficiency $(\%)$ & 62 & 32 \\
\hline Edge output $\left(\mathrm{s}^{-1}\right)$ & $1.9 \times 10^{17}$ & $1.9 \times 10^{17}$
\end{tabular}

Assuming a QT1604 solar cell dimension of $7 \mathrm{~mm} \times 7 \mathrm{~mm}$ to match the LSC photon output of $1.9 \times 10^{17} \mathrm{~s}^{-1}$ at the LSC edge, using calculations for voltage at maximum power point and radiative current from SOL. The QWSC is calculated to be operating at maximum power point MPP, at $500 \mathrm{x}$ concentration in AM1.5D light conditions.

\section{A BRIEF DISCUSSION ON OPTIMISATION}

The calibrated Monte Carlo model will be used to optimise the structure of the QWSC to enhance the waveguide efficiency to the values in Table II without loss of cell power conversion efficiency. The most obvious improvement would be to remove the substrate as could be achieved in practice in a lift off process. This would be predicted to give a significant increase in edge emission due to the removal of what is essentially a photon sink. Solar cell structures could be optimised to further improve its waveguiding capabilities. Due to having a high refractive index, a solar cell has the ability to act as a good LSC if other free carrier absorption is minimised. For example, adding a second lower-refractive index window layers into a structure would enhance transverse waveguiding to the edges. If these optical confining structures are doped for as to provide the built-in field there need not be any intentional doping in the GaAs and quantum well active region. Lessening the dimensions of the solar cell would further reduce the free carrier absorption rate. Further studies on the Monte Carlo model will lead to the identification of better waveguide designs.

To increase the optical efficiency of an LSC, progress needs to be made on novel luminescent materials. Recent challenges involve striking a balance between attempting to engineer a larger Stokes shift between the absorption and emission spectra of the luminophores in order to decrease reabsorption losses, without decreasing the luminescent quantum yields.

Wavelength selective mirrors present a bottleneck in the system performance due to the non-ideal reflection behaviour that occurs at large angles of incidence. A point to note is that this could be mitigated in part by the development of novel luminescent materials, especially in the design of anisotropic emission. Research is currently being undertaken in this area [13]. Hot and cold mirrors from Edmund Optics were purchased and a characteristion was performed of their reflectivities as a function of angle and wavelength. Results are shown in Figure 8

\section{Conclusions}

The authors have presented a novel way to manage radiative losses from a quantum well solar cell (QWSC) and diffuse light, with the objective being to power a doubly-illuminated photoelectrochemical reactor to produce $\mathrm{H}_{2}$. A QWSC is embedded in a luminescent solar concentrator (LSC), where light generated from radiative recombination escapes from the edges of the QWSC and trapped in the LSC through total internal reflection. The LSC absorbs diffuse light and traps re-emitted light. Wavelength selective mirrors are used to direct these two photon streams to their desired output edge. The design of the QWSC is a critical factor in enabling the efficicient waveguiding and transmission of the radiative light out of its edges. Raytrace modelling from an in-house raytracer will be used to determine the performance of the whole optical setup.

\section{REFERENCES}

[1] A. Fujishima \& K. Honda, "Electrochemical photolysis of water at a semiconductor electrode", Nature, 238, pp 37-28, 1972

[2] L. Minggu et al, "An overview of photocells and photoreactors for photoelectrochemical water splitting", Int. J. Hydrogen En., vol. 35, 11, 2010

[3] U.S. Department of Energy. Hydrogen, fuel cells \& infrastructure technologies program. Hydrogen production. DOE/GO-102007-2430. p.3.1 $-20$

[4] S. Sze \& K. Ng, Physics of semiconductor devices, 3rd ed. 
[5] Gueymard et al, "Proposed reference irradiance spectra for solar energy systems testing", Solar Energy, vol 73, 6, 2002

[6] D.J. Farrell, pvtrace Optical ray tracing for photovoltaic devices and luminescent materials, https://github.com/danieljfarrell/pvtrace

[7] Gueymard, "SMARTS, A Simple Model of the Atmospheric Radiative Transfer of Sunshine: Algorithms and Performance Assessment", Technical Report No. FSEC-PF-270-95. Cocoa, FL: Florida Solar Energy Center, 1995

[8] K.W.J. Barnham and G.Duggan, A new approach to high-efficiency multiband gap solar cells,J. Applied Physics, vol. 67, pp. 3490-3493, 1990

[9] D.C. Johnson, I.M. Ballard, K.W.J. Barnham, J.P. Connolly, J.S. Roberts, G. Hill, R. Airey and M. Mazzer, Obervation of photon recycling in strainbalanced quantum well solar cells, App. Phys. Lett., vol. 90, 213505, 2007

[10] K-H. Lee, K.W.J Barnham, J.P. Connolly, B.C Browne, R.J. Airey, J.S. Roberts, M. Fuhrer, T.N.D. Tibbits, N.J. Ekins-Daukes, "Demonstration of Photon Coupling in Dual Multiple-Quantum-Well Solar Cells", in IEEE J. Photovolt., vol.2, pp.68,74, 2012

[11] N.J. Ekins-Daukes, K-H. Lee, L. Hirst, A. Chan, M. Fuhrer, J. Adams, B. Browne, K.W.J. Barnham, P. Stavrinou, J. Connolly, J.S. Roberts, B. Stevens, R. Airey and K. Kennedy, Controlling radiative loss in quantum well solar cells, J. Phys. D: Appl. Phys, vol. 46, 264007, 2013

[12] M. Paxman et al, "Modeling the spectral response of the quantum well solar cell." J. App. Phys. 74.1, 1993

[13] Verbunt et al, "Anisotropic light emissions in luminescent solar concentratorsisotropic systems ”, Opt. Express, 21, 2013 